\title{
Transport of Triplet Excitons Along Continuous 100 nm Polyfluorene Chains
}

Liang Xi, ${ }^{\dagger}$ Matthew Bird, ${ }^{\dagger}$ Gina Mauro, ${ }^{\dagger}$ Sadayuki Asaoka, ${ }^{\S}$, Andrew R. Cook, ${ }^{\dagger}$ Hung-Cheng Chen, ${ }^{\ddagger}$ and John R. Miller, ${ }^{\dagger *}$

${ }^{\dagger}$ Chemistry Department, Brookhaven National Laboratory, Upton, NY 11973

$\S$ Department of Biomolecular Engineering, Kyoto Institute of Technology, Matsugasaki, Sakyo-ku, Kyoto 606-8585

${ }^{\ddagger}$ Department of Materials Science and Engineering, Stony Brook University, Stony Brook, NY 11790

\begin{abstract}
Triplet excitons created in poly-2,7-(9,9-dihexyl)fluorene ( $\mathrm{pF}$ ) chains with end trap groups in solution are efficiently transported to and captured by the end groups. The triplets explore the entire lengths of the chains, even for $\sim 100 \mathrm{~nm}$ long chains enabling determination of the completeness of end capping. The results show that the chains continuous: they may contain transient barriers or traps, such as those from fluctuations of dihedral angles, but are free of major defects that stop motion of the triplets. Quantitative determinations are aided by the addition of a strong electron donor, TMPD, which removes absorption bands of the end-trapped triplets. For chains having at least one end trap, triplet capture is quantitative on the $1 \mu$ s timescale imposed by the use of the donor. Fractions of chains having no end traps were 0.15 for $\mathrm{pF}$ samples with anthraquinone (AQ) end traps and 0.063 with naphthylimide (NI) end traps. These determinations agreed with measurements by NMR for short ( $<40$ polymer repeat units (PRU)) chains, where NMR determinations are accurate. The results find no evidence for traps or barriers to transport of triplets, and places limits on the possible presence of defects as impenetrable barriers to less than one per 300 PRU. The present results present a paradigm different from the current consensus, derived from observations of singlet excitons, that conjugated chains are divided into "segments," perhaps by some kind of defects. For the present pF chains, the segmentation either does not apply to triplet excitons or is transient so that the defects are healed or surmounted in times much shorter than $1 \mu \mathrm{s}$. Triplets on chains without end trap groups transfer to chains with end traps on a slower time scale. Rate constants for these bimolecular triplet transfer reactions were found to increase with the length of the accepting chain, as did rate constants for triplet transfer to the chains from small molecules like biphenyl. A second set of polyfluorenes with 2-butyloctyl side chains was found to have a much lower completeness of end capping.
\end{abstract}

Keywords: Exciton transport, triplet, conjugated polymer, capping, traps, segments, barriers, traps 
Correspondence: John Miller jrmiller@bnl.gov （631) 344-4354 


\section{Introduction}

Conjugated polymers offer the promise of "molecular wires" that can easily and quickly transport electrons, holes and excitons over distances of tens or even hundreds of nm, but exciton diffusion lengths in films of conjugated polymers are typically less than $10 \mathrm{~nm}$. Some measurements of singlet excitons along isolated conjugated chains have supported the idea of long distance transport ${ }^{1-8}$, but other experiments and theory indicate that conjugated chains consist of segments or "chromophores" that are separated from each other, ${ }^{9-12}$ perhaps by some kind of defects in the chains. Keller found rapid diffusion of triplet excitons in platinum acetylide oligomer chains. ${ }^{7}$ Studies of fluorescence depolarization often support the picture of separated chromophores, ${ }^{13-16}$ but excitons appear to move rapidly in some chains ${ }^{13,17}$ and that movement can be enhanced by alignment in a liquid crystal. ${ }^{3,5}$ The picture of separated chromophores has been questioned. ${ }^{18-21}$ Still Healy's measurements of singlet exciton capture by $\mathrm{C}_{60}$ end traps on isolated polythiophene chains in solution found a diffusion length of $7 \pm 0.8 \mathrm{~nm},{ }^{22}$ pointing to hardly better transport along single chains than in films, where interchain hops may be required. Those findings lead to the question: How continuous are long, conjugated chains?

Measurements of transport of triplet excitons in our laboratory on polyfluorene chains with triplet trapping end groups pointed to highly efficient (> 90\%) capture of triplets by the end traps in chains as long as $100 \mathrm{~nm}$. The time resolution of those experiments was slow, finding the capture to occur in $<40$ ns. Two other limitations of those triplet capture experiments were 1) the end capping is incomplete, but the actual capping is not known accurately, and 2) there may be defects in the chains, but if so their numbers are not known. These two questions are coupled: one cannot be resolved without the other. While it is highly desirable to determine the rate of triplet transport, means to do that are not yet at hand due to the slow mechanism of triplet formation. Experiments described below examine the coupled questions of completeness of end capping and continuity of the chains for transport of triplet excitons.

Triplets were produced by pulse radiolysis and detected by transient absorption. The experiments use length selected poly-2,7-(9,9-dihexyl)fluorenes (pF) and pFs having anthraquinone (AQ) or naphthylimide (NI) end groups. While those end groups trap triplets, a further limitation of these methods is that the absorption spectra of the trapped triplets are similar to those of triplets in the $\mathrm{pF}$ chains ${ }^{8}$; after triplet capture an absorption band remains that is $75 \%$ (AQ) and 20\% (NI) as large as the spectrum of triplets in the pF chains. While part of those remaining absorptions may be due to triplets remaining at equilibrium in the $\mathrm{pF}$ chains because 
the traps are not deep, those remaining absorptions were nearly independent of chain length. Measurements of triplet free energies and temperature dependence determined that most of the remaining absorptions are due to end trap triplets having spectra that are weaker, but remarkably, and unexpectedly, similar to triplets in the chains. ${ }^{8}$ To alleviate this complication, present experiments use a strong electron donor, TMPD, to alter the spectra and irreversibly trap the triplets in order to unambiguously determine capping fractions.

\section{Experimental}

End-capped poly-2,7-(9,9-dihexylfluorenes) (pFA) were prepared by polycondensation of 2,7-dibromo-9,9-dihexylfluorene according to the procedure reported by Klaerner and Miller $^{23}$ and by Asaoka. ${ }^{24}$ The pFAs (Chart 1 ) were then fractionally separated with preparative scale GPC into fractions with narrowed length distributions as described previously. ${ }^{25}$ The shorter fractions ( $<50$ PRU) had $\mathrm{M}_{\mathrm{W}} / \mathrm{M}_{\mathrm{N}}=1.9 \pm 0.1$ and are designated by their average lengths, e.g. $\mathrm{pF}_{24} \mathrm{NI}$ for a naphthylimide-capped polymer with an average length of 24 PRU. For longer fractions $M_{W} / M_{N}$ decreased to $<1.5$. Polymers with 2-butyloctyl side chains, pBOF, pBOFNI and pBOFAQ were prepared by a similar procedure and separated into length fractions with characteristics described further below. The pBOFs

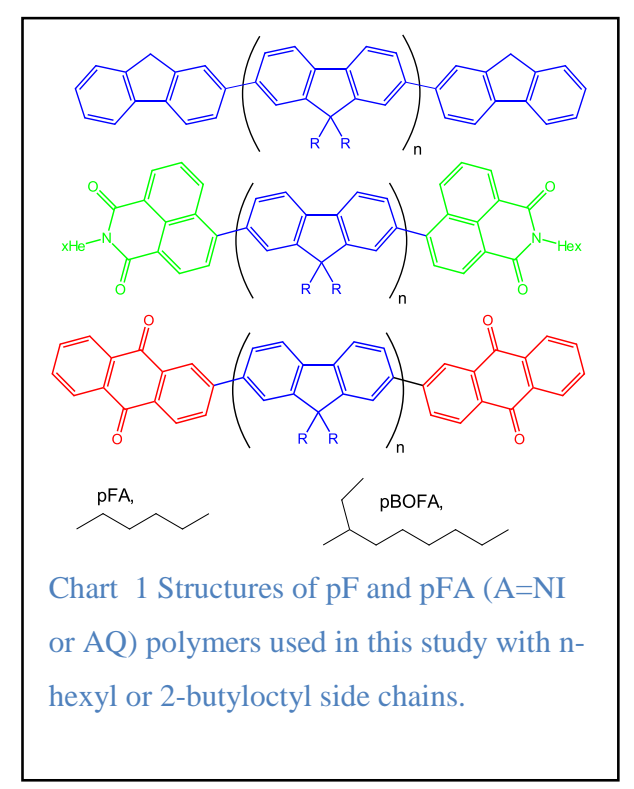
are soluble even in alkane solvents, but the synthesis gave a lower degree of end capping by the NI and AQ acceptor groups, as will also be shown below. N,N,N'N'-tetramethyl-pphenylenediamine (TMPD) from Aldrich was triply sublimed. Samples were prepared in an inert atmosphere and ionized with electron pulses from the $10 \mathrm{MeV}$ LEAF accelerator ${ }^{26}$ as described previously. ${ }^{24}$ Pulse radiolysis primarily produces ionization of the solvent, $p$-xylene or toluene, to yield electrons and radical cations, most of which recombine rapidly to produce excited states, which can then transfer to the polymers (see scheme 1). The yield of triplets is enhanced by the presence of benzophenone or acetophenone, excited states of which undergo rapid intersystem crossing to produce nearly unit yields of triplets. Naphthalene $0.1 \mathrm{M}$ was added to rapidly ( $<2 \mathrm{~ns}$ ) convert the triplets to long-lived naphthalene triplets by triplet-triplet transfer, which then transfer to the polymers. Alternatively $0.1 \mathrm{M}$ of biphenyl, reported ${ }^{27}$ to have a large (0.84) triplet quantum yield and a long (>20 $\mu$ s) lifetime, was used alone. The accelerator pulse in such solutions 
produced $\sim 1-4 \mu \mathrm{M}$ of triplets. Capture of more than one triplet on a polymer molecule would give rise to triplet-triplet annihilation, ${ }^{28-30}$ but making more than one triplet per chain is unlikely because the concentration of polymer molecules (chains) was always $>10 \mu \mathrm{M}$ and usually 50-100 $\mu \mathrm{M}$. Electronic structure calculations employed the Gaussian programs. ${ }^{31}$

\section{Results}

To learn what fraction of the chains have end traps and how many triplets reach those traps it would be desirable to remove all triplets that reach traps, while not altering triplets in $\mathrm{pF}$ chains. Table 1 makes it clear that chain and end trapped triplets are not readily distinguished on the basis of triplet energies, which are only slightly different. But reduction potentials are very different so an electron transfer process driven by the triplet energy might selectively remove trapped triplets. The table shows that the free energy to form free ions, $\mathrm{pF}^{-\bullet}+\mathrm{TMPD}^{+\bullet}$, is larger than the triplet energy of $\mathrm{pF}$ : $\mathrm{pF}$ triplets are not expected to react with TMPD by electron transfer. For both pFA molecules, pFNI and pFAQ, on the other hand this reaction is predicted to occur readily, and is thus expected to remove the strong triplet absorption bands of ${ }^{3} \mathrm{pFNI}^{*}$ and ${ }^{3} \mathrm{pFAQ}$.

Table 1 Redox potentials vs $\mathrm{Fc}^{+/ 0}$ for polymers and TMPD along with triplet free energies for $\mathrm{pF}$ chain triplets and triplets trapped by AQ or NI end groups.

\begin{tabular}{|l|l|l|l|}
\hline & $\mathrm{E}^{0} \mathrm{vs} \mathrm{Fc}^{\mathrm{a}}$ & $\Delta \mathrm{G}^{\circ}{ }_{\mathrm{T}}^{\mathrm{b}}$ & $\Delta \mathrm{G}^{\circ}{ }^{\mathrm{c}}$ \\
\hline $\mathrm{pF}^{0 /-}$ & -2.65 & 2.24 & 0.13 \\
\hline $\mathrm{pFNI}^{0 /-}$ & -1.831 & 2.18 & -0.63 \\
\hline $\mathrm{pFAQ}^{0 /-}$ & -1.437 & 2.19 & -1.03 \\
\hline $\mathrm{TMPD}^{+/ 0}$ & -0.281 & & \\
\hline biphenyl $^{\mathrm{d}}$ & -3.047 & 2.94 & \\
\hline
\end{tabular}

${ }^{a}$ Redox potentials vs ferrocene $(\mathrm{Fc})^{+/ 0}$ for the polymers ${ }^{24}$ and TMPD. ${ }^{32}$

${ }^{b}$ Triplet energies as free energy changes for formation of triplet states of $\mathrm{pF}$ chains and those end trapped on pFNI and $\mathrm{pFAQ}^{8}$ or the triplet energy of biphenyl. ${ }^{33}$

${ }^{\mathrm{c}}$ Free energy changes to form separate $\mathrm{pFA}^{-\bullet}$ and $\mathrm{TMPD}^{+\bullet}$ from ${ }^{3} \mathrm{pFA}^{*}$, where $\mathrm{A}=\mathrm{NI}$, AQ calculated from redox potentials in polar solvents containing electrolyte.

${ }^{\mathrm{d}}$ Reduction potential of biphenyl ${ }^{34}$ referred to $\mathrm{Fc}^{+/ 0}$ via anthracene from Shalev. ${ }^{35}$ 
Figure 1 shows transient absorption traces at the peak of the absorption of the pFAQ triplet. The nearly identical spectrum of pF triplet is shown in the inset. The traces with TMPD added show that the absorbing species, ${ }^{3} \mathrm{pFAQ}^{*}$, reacts with TMPD with a rate constant of $2.1 \pm 0.3 \times 10^{9} \mathrm{M}^{-1} \mathrm{~s}^{-1}$. An absorption remaining after this reaction is complete decays with a rate similar to that without TMPD (the blue trace in Figure 1). We will consider the hypothesis that this remaining absorption is due to triplets in $\mathrm{pF}$ chains having no AQ end trap groups, thus providing a measurement of how many chains lack AQ end traps, measuring the completeness of end capping. However this remaining absorption in Figure 1 depends on TMPD concentration, suggesting the presence of an equilibrium to form a coulomb bound charge transfer complex, ${ }^{3}\left(\mathrm{pFA}^{\delta-}, \mathrm{TMPD}^{\delta+}\right)$, with $\mathrm{K}_{\mathrm{eq}}=\sim 4 \times 10^{4} \mathrm{M}^{-1}$. Additional data in Figure S1 supports this conclusion.

Data in Figure 2 show that triplets in $\mathrm{pF}$ chains, ${ }^{3} \mathrm{pF}^{*}$, without end traps, decay with a $35 \mu$ s lifetime in the presence of 10mM TMPD which is indistinguishable

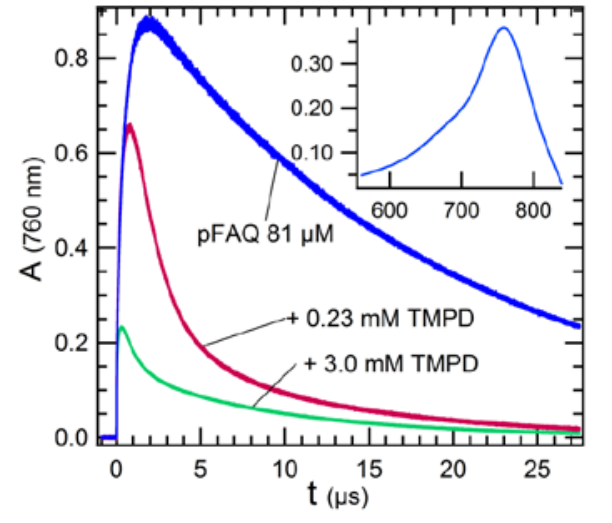

Figure 1 Transient absorption traces at the $760 \mathrm{~nm}$ absorption maximum of triplets in $81 \mu \mathrm{M}$ (polymer molecule concentration) of $\mathrm{pF}_{29} \mathrm{AQ}$ in xylene containing $100 \mathrm{mM}$ biphenyl without and with added TMPD. The inset shows the triplet band for $\mathrm{pF}+10$ mM TMPD under similar conditons.

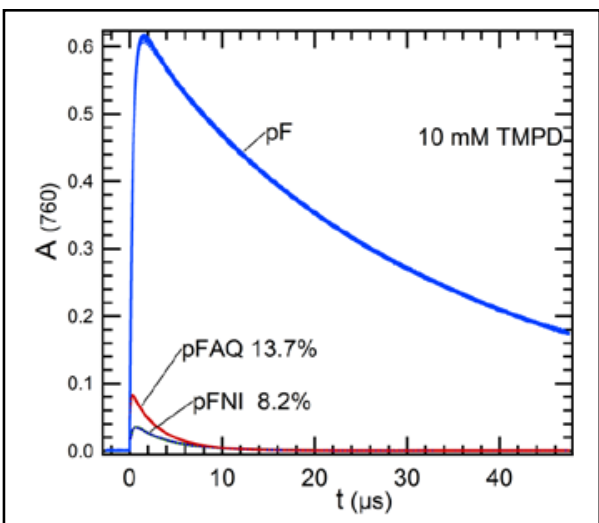

Figure 2 Transient absorption traces of $\mathrm{pF}_{28}(100 \mu \mathrm{M}), \mathrm{pF}_{22} \mathrm{NI}(100 \mu \mathrm{M})$ and $\mathrm{pF}_{20} \mathrm{AQ}(200 \mu \mathrm{M})$ in p-xylene with 100 mM biphenyl and $10 \mathrm{mM}$ TMPD.

reactions are listed in Scheme 1. With $100 \mathrm{mM}$ biphenyl (Bip) present, nearly all excited xylene the prediction, based on energies in Table 1 , that ${ }^{3} \mathrm{pF}^{*}$ does not react with TMPD. pFAQ and pFNI of similar lengths do react with TMPD. From the rate constant found in Figure 1 and the $10 \mathrm{mM}$ TMPD concentration this reaction is expected to occur with a pseudo first order rate constant of $2.1 \times 10^{7} \mathrm{~s}^{-1}$, which is slightly faster than the growths of triplet absorption for pFAQ $\left(1.1 \times 10^{7} \mathrm{~s}^{-1}\right)$ and for pFNI $\left(4.2 \times 10^{6} \mathrm{~s}^{-1}\right)$, so the rapid decays shown in Figure 1 are barely seen with the larger TMPD concentration in Figure 2. The absorptions in pFAQ and pFNI decay over a few $\mu$ s.

Reactions for forming triplets following ionization of $p$-xylene by pulse radiolysis and their subsequent

from the $38 \mu$ s lifetime found without TMPD, confirming 
triplets and singlets are converted rapidly (a few ns) to biphenyl excited states, ${ }^{3} \mathrm{Bip}^{*}$ and ${ }^{1} \mathrm{Bip}{ }^{*}$. Most recombination events will include anions and cations of Bip. A few will include polymer ions, but the resultingt excited state formation is nearly independent of which recombination reaction occurs. ${ }^{1} \mathrm{Bip}^{*}$ forms ${ }^{3} \mathrm{Bip}^{*}$ with a quantum yield of 0.86 in $\sim 16 \mathrm{~ns},{ }^{27}$ so all excited states are converted to long-lived ${ }^{3} \mathrm{Bip}^{*}$, which may then transfer its triplet excitation energy to polymers in reaction (4). Triplets were previously found to transport along chains and become trapped, reaction (5) in $<40 \mathrm{~ns}$ in chains as long as $100 \mathrm{~nm}$, so the trapping step is expected to be competitive with formation of polymer triplets, which is limited by solubility of the polymer molecules to $\sim 50 \mathrm{~ns}$.

Scheme 1: Reactions for forming and trapping triplets in p-xylene (xyl) containing $100 \mathrm{mM}$ biphenyl (Bip).

$$
\begin{aligned}
& \mathrm{xyl} \rightarrow \mathrm{xyl}^{+\bullet}+\mathrm{e}^{-} \\
& \text {(1) ionization } \\
& \mathrm{xyl}^{+\bullet}+\mathrm{e}^{-} \rightarrow{ }^{1} \mathrm{xyl}^{*}+{ }^{3} \mathrm{xyl}^{*} \quad \text { (2) recombination } \\
& { }^{3} \mathrm{xyl}^{*}+\mathrm{Bip} \rightarrow{ }^{3} \mathrm{Bip}^{*} \quad \text { (3) triplet transfer } \\
& { }^{3} \mathrm{Bip}^{*}+\mathrm{pFA} \rightarrow{ }^{3} \mathrm{pF}^{*} \mathrm{~A} \quad \text { (4) triplet transfer } \\
& { }^{3} \mathrm{pF}^{*} \mathrm{~A} \rightarrow{ }^{3} \mathrm{pFA}^{*} \quad \text { (5) triplet trapping } \\
& { }^{3} \mathrm{pFA}^{*}+\mathrm{TMPD} \leftrightharpoons{ }^{3}\left(\mathrm{pFA}^{\delta-}, \mathrm{TMPD}^{\delta+}\right)(6) \mathrm{CT} \\
& { }^{3} \mathrm{pF}^{* *}+\mathrm{pFA} \rightarrow \mathrm{pF}+{ }^{3} \mathrm{pF}^{*} \mathrm{~A} \quad \text { (7) triplet transfer }
\end{aligned}
$$

Amplitudes of the pFAQ and pFNI traces in Figure 2 are greatly reduced by reaction of the trapped triplets, ${ }^{3} \mathrm{pF}^{*} \mathrm{~A}$, with TMPD. Absorbances extrapolated to $\mathrm{t}=0$ are $13.7 \%$ (pFAQ) and $8.2 \%$ (pFNI) of the absorbance in pF without end traps. These are close to the fractions of chains having no end traps determined by NMR (see below). The pFAQ and pFNI traces also decay with rates of 3.2 and $2.5 \times 10^{5} \mathrm{~s}^{-1}$, about 10 times faster than the $3.6 \times 10^{4} \mathrm{~s}^{-1}$ decay for triplets of uncapped $\mathrm{pF}$.

These observations can be understood simply. The decaying absorbances in Figure 2 can be attributed to $\mathrm{pF}$ chains having no end traps. The fractions of uncapped chains are small. The $\mathrm{pF}$ chain triplets on these trapless chains can transfer triplets to chains having caps by bimolecular triplet transfer, reaction (7). This transfer becomes effectively irreversible due to rapid intrachain transport and trapping, reaction (5), followed by charge transfer to TMPD, reaction (6). Based on this picture and data in Figure 2 13.7\% of the $\mathrm{pF}_{20} \mathrm{AQ}$ and $8.2 \% \mathrm{pF}_{22} \mathrm{NI}$ chains have no traps. Triplets on these trapless chains transfer to chains having traps with rate constants that increase 
with chain length varying from $1.5 \times 10^{9}$ to $3.7 \times 10^{9} \mathrm{M}^{-1} \mathrm{~s}^{-1}$. We now report similar experiments with pFNIs and pFAQs with varied chain lengths.

Figure 3 plots the rate constants from uncapped to capped chains as a function of the average chain length in PRU along with similar rate constants for triplet transfer from ${ }^{3} \mathrm{Bip} *$ to $\mathrm{pF}$, pFNI and pFAQ chains. The triplet transfers from ${ }^{3} \mathrm{Bip}^{*}$ are about 20 times faster than transfers from ${ }^{3} \mathrm{pF}^{*}$. Both increase approximately linearly with chain length, as seen for electron attachment. ${ }^{25}$ The reactions of ${ }^{3} \mathrm{Bip} *$ were measured by the rate constants for the growth of polymer triplet at $760 \mathrm{~nm}$. These measurements utilized only samples without TMPD so there was only one triplet donor, ${ }^{3} \mathrm{Bip} *$, and to avoid the effects of CT to TMPD, reaction (6), which could affect the observed kinetics. Measurements of triplet transfer from ${ }^{3} \mathrm{pF}^{*}$, to ${ }^{3} \mathrm{pFA}^{*}$ used only samples with TMPD, which removes the large triplet absorptions of ${ }^{3} \mathrm{pFNI}^{*}$ or ${ }^{3} \mathrm{pFAQ}^{*}$ and renders the reaction effectively irreversible.

\section{Spectra of CT Exciplexes with TMPD When}

reaction with TMPD removes the strong absorption bands of trapped triplets, it creates new bands having shorter lifetimes. Figure 4 displays spectra after pulse

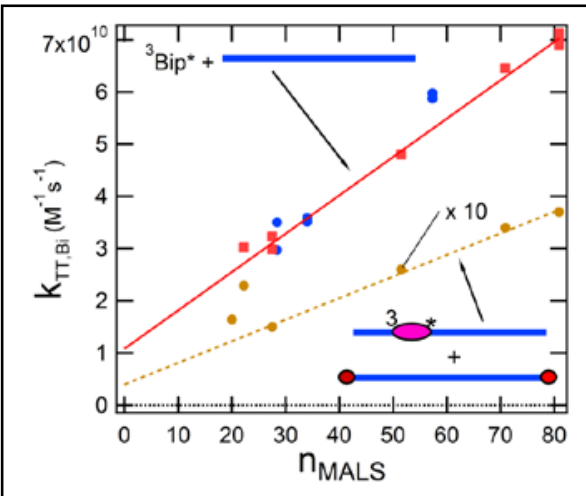

Figure 3 Rate constants for triplet energy transfer from ${ }^{3} \mathrm{Bip} *$ to $\mathrm{pF}$ (O), pFNI and pFAQ chains ( $\square$ ) vs. the average chain length. The lower points for triplet transfer from $3 \mathrm{pF}^{*}$ to $\mathrm{pFA}(\mathrm{O}, \mathrm{A}=\mathrm{NI}$ or AQ) are multiplied by 10 to show them on the same scale. radiolysis of $200 \mathrm{uM}$ pFAQ or 100uM pFNI reacting with 10mM TMPD. The triplet absorption bands at $760 \mathrm{~nm}$ are largely replaced by bands peaking near $590 \mathrm{~nm}$. After $100 \mathrm{~ns}$ we attribute these bands mainly to complexes formed by reaction (6) that we refer to as ${ }^{3}\left(\mathrm{pFA}^{\delta-}, \mathrm{TMPD}^{\delta+}\right)$, where the degree of charge transfer, $\delta$, is not clear. Transient absorption traces in Figure 4c) show that ${ }^{3}\left(\mathrm{pFAQ}^{\delta-}, \mathrm{TMPD}^{\delta+}\right)$ has a lifetime of $0.24 \pm 0.05 \mu$ s while ${ }^{3}\left(\mathrm{pFNI}^{\delta-}, \mathrm{TMPD}^{\delta+}\right)$ has a lifetime of $1.13 \pm 0.3 \mu \mathrm{s}$. A shorter lived (42 ns) species is evident in the pFNI trace, signaling that geminate ion recombination is taking place on this time scale and that some of the ions recombining in reaction (3) are $\mathrm{pF}$ ions, $\mathrm{known}^{24}$ to absorb at $\sim 590 \mathrm{~nm}$. A fit of the pFAQ trace in Figure 4c) finds a similar (37 ns) lifetime component with a similar amplitude, although it does not stand out as clearly due to the reasonably rapid $(0.24 \mu \mathrm{s})$ decay of ${ }^{3}\left(\mathrm{pFAQ}^{\delta-}, \mathrm{TMPD}^{\delta+}\right)$, absorbing at the same wavelength. The spectrum of ${ }^{3}\left(\mathrm{pFNI}^{\delta-}, \mathrm{TMPD}^{\delta+}\right)$ has a low energy band peaking at $960 \mathrm{~nm}(1.3 \mathrm{eV})$. This band grows, reaches a maximum at $~ 500 \mathrm{~ns}$ and decays with little interference from ions or triplets in uncapped $\mathrm{pF}$ chains. Its lifetime, $1.4 \mu$ s, is similar 
to the $1.13 \mu$ s lifetime at $760 \mathrm{~nm}$, but is probably more accurate. The energy of this band is close to the $1.55 \mathrm{~V}$ difference in reduction potentials of pFNI and $\mathrm{TMPD}^{+\bullet}$ in Table 1 , suggesting that it could be a charge transfer band from TMPD to $\mathrm{pFNI}:{ }^{3}\left(\mathrm{pFNI}^{\delta-}, \mathrm{TMPD}^{\delta+}\right) \rightarrow^{3}\left(\mathrm{pFNI}^{(1-\delta)-}, \mathrm{TMPD}^{(1-}\right.$ ${ }^{\delta)^{+}}$), where $\delta<<1$. A similar band for pFAQ could be predicted from the potentials to occur at $\sim 1500 \mathrm{~nm}$. While that region was not examined, a small spectral component with its lifetime is seen from 850-1000 nm. As noted above, the growths at $760 \mathrm{~nm}$ in pFNI and pFAQ are due primarily to transfer of triplets from ${ }^{3} \mathrm{Bip} *$ to uncapped chains. 

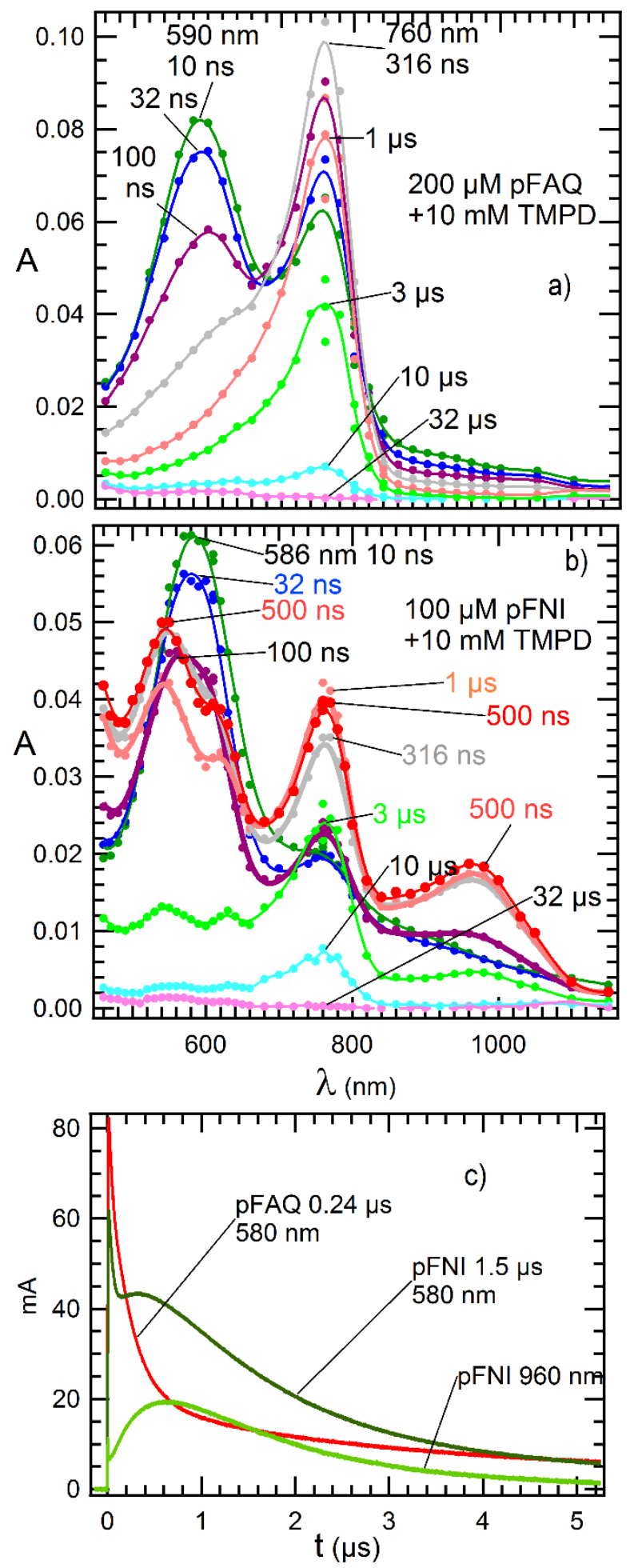

Figure 4 Spectra of transient species formed after pulse of a) $200 \mu \mathrm{M} \mathrm{pF}_{20} \mathrm{AQ}$ or b) $100 \mu \mathrm{M} \mathrm{pF}_{22} \mathrm{NI}$, both with $10 \mathrm{mM}$ TMPD in p-xylene containing $100 \mathrm{mM}$ biphenyl. c) transient absorption traces at $580 \mathrm{~nm}$ and $960 \mathrm{~nm}$. 
Completeness of End Capping by Triplet Trapping with TMPD Triplet capture with suppression of end trap triplet absorption by TMPD provides a method to determine the completeness of end capping. While a low concentration of TMPD, like the $0.23 \mathrm{mM}$ used in one trace in Figure 1, reacts sufficiently rapidly, a larger concentration is needed to push the equilibrium of reaction (6) to completion. For $K_{e q}=4 \times 10^{4}$ $\mathrm{M}^{-1}$, estimated above for reaction 6, $10 \mathrm{mM}$ TMPD would leave only $0.25 \%$ of the polymer triplet, enabling a measurement of the remaining absorbance from chains having no trap groups. Figure S2 gives an example in which a large concentration of TMPD is used. In this example, the concentration of polymer is low $(\mu \mathrm{M})$, so there is little triplet transfer from uncapped $\mathrm{pF}$ chains to chains with traps. In that case, the absorbance with TMPD, multiplied by a scale factor, is nearly identical to the absorbance trace in $\mathrm{pF}+\mathrm{TMPD}$, providing some verification of the method. This measurement yields the fraction, $F_{0}$, of chains having no trap groups. The triplet charge transfer species with TMPD give little absorption at $760 \mathrm{~nm}$ and thus little interference with the measurement of remaining triplets on chains without end traps. Due to the short lifetimes of the charge transfer species, good accuracy in determination of $F_{0}$ was obtained by utilizing data after they had decayed.

Figure 5 graphs results from both triplet capture with TMPD and from measurements by NMR described below. The filled points are from triplet capture with TMPD; the open squares are from NMR. For short $(n<40$ PRU) chains where NMR measurements are accurate there is reasonable agreement between NMR and triplet/TMPD determinations of the fraction of chains without trap groups. At longer lengths the triplet/TMPD method may remain reasonably accurate. Line fits to the triplet/TMPD data in Figure 5 give $F_{0}=0.148$ for $\mathrm{pFAQ}$ and $F_{0}=0.0625$ for $\mathrm{pFNI}$, assuming $F_{0}$ to be independent

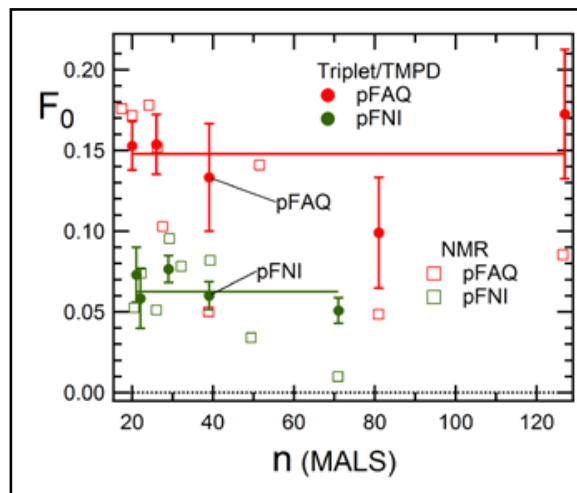

Figure 5 Fractions of chains having no end trap groups, $\mathrm{F}_{0}$, measured by triplet capture with TMPD $(\bullet$ pFNI, $\bullet$ pFAQ $)$ and by NMR ( $\square$ pFNI, $\square$ pFAQ), as a function of the average lengths of the separated polymer fractions. Line fit to the data give $\mathrm{F}_{0}=0.148$ (pFAQ) and 0.0625(pFNI). Probability of end capping $=P_{\text {cap }}=0.615$ and 0.75 respectively.

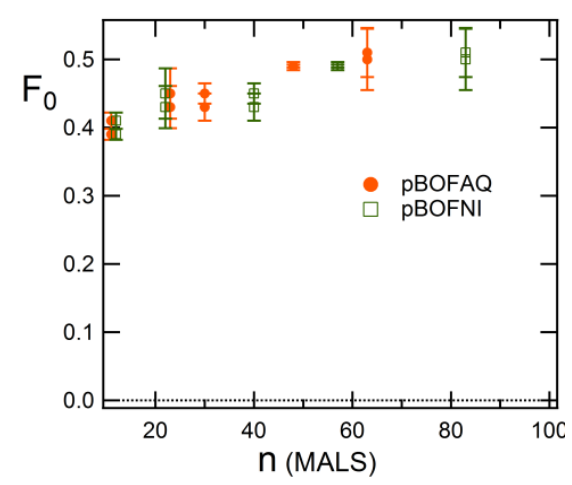

Figure 6 Fractions of poly(2-butyloctyl)fluorene polymers having no NI or AQ end traps determined by the triplet/TMPD method, graphed vs. the average length of the fractions. 
of chain length. Within the noise of the measurements, due to pulse-to-pule instability, no trend is observed in which $F_{0}$ depends on chain length. The data also provide evidence against the presence of substantial numbers of defects as will be discussed below.

Measurements of end trap incorporation in the pBOF polymers are shown in Figure 6. From this data it is clear that the degree of end capping is much lower than with the polymers having hexyl side chains. There is also no difference between the degree of capping for the NI and $\mathrm{AQ}$ end groups. For both end groups the fraction without traps, $F_{0}$, appears to increase slightly with increasing length, but this increase is close to uncertainties of the measurements. For $F_{0}=0.45$, an average over lengths from data in Figure 6, the probability that a chain end is capped, $P_{\text {cap }}$, is 0.33 , thus the fraction of chains with 1 and 2 caps are $F_{1}=0.44$ and $F_{2}=0.11$ respectively: only $11 \%$ of the chains have the desired two end trap groups.

Completeness of End Capping by NMR The completeness of end capping can also be measured by NMR using integrations of prominent lines of the NI or AQ end groups and lines from the hexyl side chains of the pFs. Results are given in Table S1, which gives a name for each fraction, its average length in PRU and the probability, $P_{\text {cap }}$, that a chain end has an NI or AQ trap group. The fractions, separated by GPC, have much narrower length distributions than the original polymers but there is still a substantial range of lengths within each fraction. While the triplet/TMPD measurements give directly an averaged value of the fraction of chains having no end traps, the NMR just gives the relative concentrations of trap groups and $\mathrm{pF}$ monomers. Within a given polymer fraction, shorter chains require more end trap groups per $\mathrm{pF}$ monomer to achieve a given degree of capping. Therefore, to calculate $P_{c a p}$, it is necessary to account for the distribution of lengths in each fraction. To do so, the NMR data was expressed as the ratio of “monomers (repeat units) per 2 caps.” Next,considering that each chain has two ends, the number of " 2 ends" per monomer repeat unit was found, averaged over the distribution of lengths. This is equivalent to finding the average of the reciprocal of the polymer length $\langle 1 / n\rangle$. The following integral was evaluated for each of the narrowed length fractions in Table S1, using the relevant normalized number distribution, $p(n)$, of the polymer lengths:

$$
\text { "2 ends" per monomer }=\langle 1 / n\rangle=\int_{0}^{\infty} \frac{1}{n} p(n) d n
$$

Finally, the probability of an end being capped, $P_{c a p}$, can be found by the ratio:

$$
P_{\text {cap }}=\left(\frac{\text { caps }}{\text { end }}\right)=\frac{1}{\left(\frac{\text { "2 ends" }}{\text { monomer }}\right) \times\left(\frac{\text { monomers }}{2 \text { caps }}\right)}
$$

The fractions of polymers capped with zero $\left(F_{0}\right)$, one $\left(F_{1}\right)$, or two $\left(F_{2}\right)$ end trap groups could then be derived from this fraction as 
$F_{0}=\left(1-P_{c a p}\right)^{2}, F_{1}=2 P_{c a p}\left(1-P_{c a p}\right), F_{2}=P_{c a p}{ }^{2}$.

Eqs 3 assumes that during synthesis reactions occurring at one end of the chain are independent of reactions at the other end. The results find that for shorter fractions $P_{\text {cap }}$ is approximately constant, but they differ for pFNI and pFAQ. Average values are $\left\langle P_{\text {cap }}\right\rangle(\mathrm{pFNI})=0.73$ and $<P_{c a p}>(\mathrm{pFAQ})=0.63$ from the shorter fractions. In fractions with lengths $>50$ PRU the NMR is inaccurate, as expected, because the signals from the end groups are very small.

\section{Discussion}

Data presented here find that triplet excited states of AQ and NI end capped polymers react with TMPD to form excited complexes with TMPD. Formation of the complexes removes the strong absorption bands of the end-trapped triplets, yielding measurements of the extent of end-capping, the ability of triplets to migrate along the chains, and rate constants for triplet energy transfer from one chain to another.

Natures of the complexes The absorption bands formed upon reaction of ${ }^{3} \mathrm{pFA}^{*}$ with TMPD were referred to as charge transfer species ${ }^{3}\left(\mathrm{pFAQ}^{\delta-}, \mathrm{TMPD}^{\delta+}\right)$ and ${ }^{3}\left(\mathrm{pFNI}^{\delta-}, \mathrm{TMPD}^{\delta^{+}}\right)$. In support of this interpretation both have broad, strong bands peaking at $590 \mathrm{~nm}$ and the pFNI complex displays another broad band at $960 \mathrm{~nm}$. The observations that uncapped ${ }^{3} \mathrm{pF}^{*}$ does not react with TMPD, while the ${ }^{3} \mathrm{pFA}^{*}$ molecules do, correlates with redox potentials (Table 1 ) to implicate a charge transfer reaction, as proposed in reaction (6). The observation of equilibrium in reaction (6) might indicate that the degree of charge transfer is small in the very weakly polar $p$-xylene environment. Of the two complexes the degree of charge transfer would be expected to be larger for ${ }^{3}\left(\mathrm{pFAQ}^{\delta-}, \mathrm{TMPD}^{\delta+}\right)$ due to the more positive redox potential of pFAQ (Table 1 ), so the lifetime of ${ }^{3}\left(\mathrm{pFAQ}^{\delta-}, \mathrm{TMPD}^{\delta+}\right)$ would be expected to be shorter, as observed.

Bimolecular Triplet Energy Transfer Reactions The rate constants for triplet transfer (TT) from ${ }^{3} \mathrm{Bip} *$ to $\mathrm{pF}_{\mathrm{n}}, \mathrm{pF}_{\mathrm{n}} \mathrm{AQ}$ and $\mathrm{pF}_{\mathrm{n}} \mathrm{NI}$, reaction (4), increase approximately linearly with chain length. The rates are similar for transfer to $\mathrm{pF}$ and to $\mathrm{pFAs}$ : triplet transfer appears to occur principally to the long polymer chains. There may be a contribution from transfer directly to the end trap groups, but it is not dominant. For longer chains the rate constants reach $7 \times 10^{10} \mathrm{M}^{-1} \mathrm{~s}^{-1}$. This is much faster than triplet transfer rates to small molecules, which reach rate constants of almost $1 \times 10^{10} \mathrm{M}^{-1} \mathrm{~s}^{-1} .{ }^{33}$ The apparently linear increase of $\mathrm{k}\left({ }^{3} \mathrm{Bip} *+\mathrm{pF}\right)$ with chain length has an intercept of $1 \times 10^{10} \mathrm{M}^{-1} \mathrm{~s}^{-1}$. The intercept could include a contribution from direct transfer from ${ }^{3} \mathrm{Bip} *$ to the end groups, or could signal that the increase is not linear at shorter lengths. While approximately linear, the increase is not proportional to the $\mathrm{pF}$ length; from $n=20-80$ PRU the 
length increases by a factor of 4 , but the rate constant increases by a factor of 2.3. This behavior is expected if the TT reactions to the $\mathrm{pF}$ chains are diffusion-controlled, or nearly so. Indeed a diffusion-controlled reaction might be expected for $\Delta \mathrm{G}^{\circ}=-0.7 \mathrm{eV}$ from triplet energies in Table 1 . The slope of the linear fit in Figure 3 is $7.3 \times 10^{8} \mathrm{M}^{-1} \mathrm{~s}^{-1}$ per repeat unit, which is smaller by a factor of $\sim 10$ than diffusion controlled reactions by small molecules. This rate per repeat unit may reflect “diffusive interaction. ${ }^{„ 36,37}$ Diffusive interaction occurs when rapid reaction of a diffusing species, such as ${ }^{3} \mathrm{Bip} *$, with a long chain reactant removes the ${ }^{3} \mathrm{Bip} *$, preventing later encounters with the chain from occurring. The behavior observed here mirrors the observation of Sreearunothai ${ }^{25}$ who measured rate constants for attachment of solvated electrons to $\mathrm{pF}$ chains of increasing lengths. For chains longer than $\sim 20$ PRU the rate constant per repeat unit was $\sim 1 / 10$ of diffusion-controlled rates to small molecules. ${ }^{25}$

Rate constants from ${ }^{3} \mathrm{pF}^{*}$ to pFNI or pFAQ in Figure 3 are slower by a factor of $\sim 20$ than rate constants from ${ }^{3} \mathrm{Bip} *$. While there is scatter, these rate constants also appear to increase with increasing chain length, again suggesting that the transfer occurs mainly to $\mathrm{pF}$ chains, followed by triplet transport to the AQ or NI end trap, reaction (5) and reaction with TMPD, reaction (6), which renders the reaction substantially irreversible for sufficiently large ( $>\sim 3 \mathrm{mM}$ ) TMPD concentrations. The rate constants do not depend on which end group is present, again supporting chain to chain transfer as the principal mechanism. They are slower than reactions for triplet transfer from ${ }^{3} \mathrm{Bip} *$ due to slower diffusion of $\mathrm{pF}$ chains and because $\Delta \mathrm{G}^{\circ}=0$ for the rate determining step, reaction (5), which transfers a triplet from one $\mathrm{pF}$ chain to another. Diffusion coefficients for polymers from 20-80 PRU decrease by factors of $~ 5-10$ relative to those of small molecules based on diffusion measurements by Fytas and Somma. ${ }^{38,39}$ The observed decrease of the ${ }^{3} \mathrm{pF}^{*}+\mathrm{pFA}$ rate constants by a factor of $\sim 20$ relative to triplet transfer from ${ }^{3} \mathrm{Bip} *$ therefore suggests that the ${ }^{3} \mathrm{pF}^{*}+\mathrm{pFA}$ rates are below diffusion control by a factor of $\sim 2-4$ due to the zero free energy change. Within the uncertainties of the measurements, these slower rate constants are close to proportional to the lengths of the pFNI and pFAQ chains. For reactions that are not diffusion-controlled the rate constants for transfer to different portions of the accepting chain should add linearly so that the rate constant for transfer to the whole chain would be proportional to the lengths of the chains.

Completeness of End Capping, Continuity of Chains and Defects The fractions, $F_{0}$, of chains having no end traps, determined by triplet trapping with TMPD, is identical within experimental uncertainty to $F_{0}$ estimated from NMR/MALS for short chains, where the NMR determinations can be accurately made. The measurements indicate that the fractions of chains without end traps are $0.148 \pm 0.02$ for all pFAQ fractions and $0.0625 \pm 0.02$ for all pFNI fractions measured (up to 
$\mathrm{n}=70 \mathrm{PRU})$. The data appear to rule out a strong trend of $F_{0}$ increasing or decreasing with chain length. While a weak trend is possible, the data are most consistent with constant $F_{0}$, and therefore constant degree of end capping. The agreement of triplet/TMPD and NMR measurements suggests that if a chain has at least one AQ or NI end group, a triplet exciton on the chain will find it within the time scale of these measurements, $\sim 1 \mu \mathrm{s}$. The fractions of chains with 0,1 or 2 end trap groups are given in Table 2 .

Table 2 Fractions of polyfluorenes, having zero, one and two NI or AQ end traps. pFNI and pFAQ have hexyl side chains. pFBOFA chains, with 2-butyloctyl side chains, have similar capping fractions with NI or AQ end groups.

\begin{tabular}{lrrr} 
& \multicolumn{1}{c}{ pFNI } & pFAQ & pFBOFA \\
$F_{0}$ & 0.07 & 0.14 & 0.45 \\
$F_{1}$ & 0.39 & 0.47 & 0.44 \\
$F_{2}$ & 0.53 & 0.39 & 0.11
\end{tabular}

An important possibility examined by this data is that the chains contain defects. Defects would be expected to occur randomly, so analysis below will be based on the assumption that the probability of a defect is proportional to the length of the chain. Such defects could act as traps that destroy triplet excitons, as traps that immobilize them, or as barriers that prevent their passage. The present data offers evidence against all three types, for moderately high barriers or moderately deep traps. Barriers or immobilizing traps, if present, would have prevented triplet capture by NI or AQ end groups, thus increasing the apparent $F_{0}$ in longer chains. Such an increase does not occur. Figure S2 shows the data of Figure 5 again and includes fit curves for a model that includes randomly placed barrier defects at densities, $\rho$, of 0.001-0.01 barriers per repeat unit. The barriers are impenetrable. The model is described in text accompanying Figure S2. A curve with one barrier defect every 1000 PRU $(\rho=0.001)$ is indistinguishable from the pFAQ data, but the model curve for one barrier every 300 PRU departs substantially. If present at all, there is less than one such defect per 300 PRU. For pFNIs, the limit is less stringent, less than one barrier for each 150 PRU because molecules with lengths > 80 PRU were not available. Traps that immobilize triplets, but retain their spectra would also increase the apparent $F_{0}$ in chains having such traps. The increase would be slightly larger than that for barrier defects, so the limits set by the data are at least as stringent. 
The present experiments do not exclude the possibility of barriers or traps that are shallow or of a transient nature. These could include transient barriers (or traps) created by unfavorable (or favorable) dihedral angles that might easily improve within the $\sim 1 \mu$ s time resolution of the present experiments..Such a picture seems to be suggested in a simulation of Willard and Rossky. ${ }^{40}$ Mani $^{41}$ recently found that unfavorable dihedral angles can serve as barriers for motion of electrons, holes or excitons, but presented the largest barriers for motion of singlet excitons. Relaxation of unfavorable dihedral angles and Mani's findings may together explain the apparent difference between efficient triplet transport reported here as compared to confinement of singlet excitons to segments. The present experiments were carried out on conjugated chains in liquids. If transient barriers or traps occur and heal within our time resolution, they might not so readily do so in solid films.

Evidence for deep traps that remove or destroy triplets is also absent. The observation of $\mathrm{pF}$ triplets tells us that such destructive traps are present at low densities, if at all. The results with pFNIs or pFAQs with TMPD tell us that triplets explore the entire chains, so a triplet-destroying defect anywhere in a pF chain would remove triplets from that chain. Because defects would be more likely in long chains, the GPC pF fractions having long average chain lengths should have lower triplet yields. If, for example, there is one destructive defect for each 50 PRU, then triplets would survive in $82 \%$ of chains 20 PRU in length, but only 13\% would survive in chains 100 PRU in length. Data in Figure S3 find less than 15\% difference in triplet yields in pBOF chains from 15 to 165 PRU, pointing to a deep defect density $\rho<0.002$ per repeat unit, which is similar to the findings for barrier defects. A simple summary is that the data find no evidence for defects that can act as deep traps or large barriers that cannot be surpassed within $1 \mu \mathrm{s}$. The upper limits set for all such major defects are one per 300 PRU.

An alternate possibility could explain some of the results. Because the polymer chains are in solution they could fold back on themselves and come into contact. Such self-contacts could transfer triplets from portions of a chain that are isolated from end traps by barrier defects. Figure S6 pictures such a possibility for a chain with one end trap group. Short chains cannot easily curl sufficiently to make contact as indicated by the 10 PRU persistence length of polyfluorenes, ${ }^{42}$ so the present conclusions still apply to the shorter chains. If only data from short chains is used, the limits on defects are less stringent. If triplet transfer via self-contacts occur readily for longer chains, that mechanism might partially vitiate the conclusion that chains are defect-free to very long lengths. An estimate of the rate constant for triplet transfer via self-contacts, given in figure S7, treats a 100 PRU long chain having one end trap as two 50 PRU chains, one having an end trap. These chains diffuse freely within the confines of a cubic box with a volume defined by the 
average size of a one 100 PRU chain. The estimate, described below Figure S7, utilizes the chain to chain bimolecular triplet transfer rate constants reported in Figure 3. It finds that triplet transfer via self-contacts occurs more slowly than the $1 \mu$ s time resolution of the present experiments, and much more slowly than the 40 ns resolution reported earlier by Sreearunothai ${ }^{8}$ for $\mathrm{pFNI}$ without TMPD. Detailed knowledge of the conformational dynamics of a single chain and the competition between intra and intermolecular triplet transfer rates during a single close chain encounter would be required for a full theoretical treatment. However, the estimate of Figure S7, for two confined half chains, which uses appropriate measured triplet transfer rates, provides reasonable evidence that triplet transfer, via self-contact, to bypass defects on the time scale of this experiment are likely negligible, strengthening the conclusion that the chains are highly defect free.

\section{Conclusions}

In polyfluorenes with anthraquinone (AQ) or naphthylimide (NI) end trap groups, triplets created in the chains transport to and are captured by the end groups. For chains up to $100 \mathrm{~nm}$ in length, triplet capture is quantitative for all chains with at least one end trap group in experiments with $1 \mu$ s time resolution, indicating the triplet excitons explore the entire lengths of the chains.

The present data finds no evidence for large traps or barriers to motion of triplets in these pF polymers. On the contrary they set limits that there are fewer than one such defect per 300 repeat units. The present experiments do show that the while many experimental results on singlet excitons point to a picture in which conjugated chains consist of separate chromophores, the barriers between those chromophores must not be static or perhaps do not apply to triplets in these $\mathrm{pF}$ chains. The results also tell us that transport of triplet excitons should be explored further. In the present experiments do not find evidence that triplet transport is interrupted by defects, but if, as earlier results suggest, triplet transport is much faster than the present $\sim 1 \mu \mathrm{s}$ time scale, ${ }^{7,8}$ then transport may be briefly interrupted but succeed later. Efforts to examine triplet transport on faster time scales may be important. The present experiments do not exclude the possibilities that shallow traps or barriers may exist for short times or that other conjugated polymers may contain substantial traps or barriers. PPVs are known to contain defects which shape their conformations. ${ }^{43-45}$ 
Acknowledgment: This material is based upon work supported by the U.S. Department of Energy, Office of Science, Office of Basic Energy Sciences through Grant DE-AC02-98CH10886, including use of the LEAF and Van de Graaff facilities of the BNL Accelerator Center for Energy Research. We thank Alexis Estrada for assistance in collecting data in Figure S1.

Supporting Information Available: Graphs showing kinetics of triplet reaction and models of defects along with description of the model, tables of NMR estimates of end capping, triplet absorption as a function of length, and a model and results for self-contacts.This material is available free of charge via the Internet at http://pubs.acs.org.

\section{References}

(1) Swager, T. M.; Gil, C. J.; Wrighton, M. S., Fluorescence Studies of Poly(PPhenyleneethynylene)S - the Effect of Anthracene Substitution, J. Phys. Chem. 1995, 99, 48864893.

(2) Tan, C. Y.; Alas, E.; Muller, J. G.; Pinto, M. R.; Kleiman, V. D.; Schanze, K. S., Amplified Quenching of a Conjugated Polyelectrolyte by Cyanine Dyes, J. Am. Chem. Soc. 2004, 126, 13685-13694.

(3) Nesterov, E. E.; Zhu, Z. G.; Swager, T. M., Conjugation Enhancement of Intramolecular Exciton Migration in Poly(P-Phenylene Ethynylene)S, J. Am. Chem. Soc. 2005, 127, 1008310088.

(4) Funston, A. M.; Silverman, E. E.; Schanze, K. S.; Miller, J. R., Spectroscopy and Transport of the Triplet Exciton in a Terthiophene End-Capped Poly(Phenylene Ethynylene), J. Phys. Chem. B 2006, 110, 17736-17742.

(5) Rose, A.; Tovar, J. D.; Yamaguchi, S.; Nesterov, E. E.; Zhu, Z. G.; Swager, T. M., Energy Migration in Conjugated Polymers: The Role of Molecular Structure, Philosophical Transactions of the Royal Society a-Mathematical Physical and Engineering Sciences 2007, 365, 1589-1606.

(6) Shibano, Y.; Imahori, H.; Sreearunothai, P.; Cook, A. R.; Miller, J. R., Conjugated "Molecular Wire" for Excitons, J.Phys. Chem. Lett. 2010, 1, 1492-1496.

(7) Keller, J. M.; Glusac, K. D.; Danilov, E. O.; McIlroy, S.; Sreearuothai, P.; R. Cook, A.; Jiang, H.; Miller, J. R.; Schanze, K. S., Negative Polaron and Triplet Exciton Diffusion in Organometallic "Molecular Wires", J. Am. Chem. Soc. 2011, 133, 11289-11298.

(8) Sreearunothai, P.; Estrada, A.; Asaoka, S.; Kowalczyk, M.; Jang, S.; Cook, A. R.; Preses, J. M.; Miller, J. R., Triplet Transport to and Trapping by Acceptor End Groups on Conjugated Polyfluorene Chains, J. Phys. Chem. C 2011, 115, 19569-19577. 
(9) Hwang, I.; Scholes, G. D., Electronic Energy Transfer and Quantum-Coherence in PiConjugated Polymers, Chem. Mater. 2011, 23, 610-620.

(10) Dykstra, T. E.; Hennebicq, E.; Beljonne, D.; Gierschner, J.; Claudio, G.; Bittner, E. R.; Knoester, J.; Scholes, G. D., Conformational Disorder and Ultrafast Exciton Relaxation in PpvFamily Conjugated Polymers, J. Phys. Chem. B 2009, 113, 656-667.

(11) Hennebicq, E.; Pourtois, G.; Scholes, G. D.; Herz, L. M.; Russell, D. M.; Silva, C.; Setayesh, S.; Grimsdale, A. C.; Mullen, K.; Bredas, J. L.et. al., Exciton Migration in Rigid-Rod Conjugated Polymers: An Improved Forster Model, J. Am. Chem. Soc. 2005, 127, 4744-4762.

(12) Beljonne, D.; Pourtois, G.; Silva, C.; Hennebicq, E.; Herz, L. M.; Friend, R. H.; Scholes, G. D.; Setayesh, S.; Mullen, K.; Bredas, J. L., Interchain Vs. Intrachain Energy Transfer in Acceptor-Capped Conjugated Polymers, Proc. Nat. Acad. Sci. USA 2002, 99, 10982-10987.

(13) Muller, J. G.; Lupton, J. M.; Feldmann, J.; Lemmer, U.; Scherf, U., Ultrafast Intramolecular Energy Transfer in Single Conjugated Polymer Chains Probed by Polarized Single Chromophore Spectroscopy, Appl. Phys. Lett. 2004, 84, 1183-1185.

(14) Ruseckas, A.; Wood, P.; Samuel, I. D. W.; Webster, G. R.; Mitchell, W. J.; Burn, P. L.; Sundstrom, V., Ultrafast Depolarization of the Fluorescence in a Conjugated Polymer, Phys. Rev. B 2005, 72.

(15) Collini, E.; Scholes, G. D., Coherent Intrachain Energy Migration in a Conjugated Polymer at Room Temperature, Science 2009, 323, 369-373.

(16) Mirzov, O.; Bloem, R.; Hania, P. R.; Thomsson, D.; Lin, H. Z.; Scheblykin, I. G., Polarization Portraits of Single Multichromophoric Systems: Visualizing Conformation and Energy Transfer, Small 2009, 5, 1877-1888.

(17) Da Como, E.; Borys, N. J.; Strohriegl, P.; Walter, M. J.; Lupton, J. M., Formation of a Defect-Free Pi-Electron System in Single Beta-Phase Polyfluorene Chains, J. Am. Chem. Soc.

2011, 133, 3690-3692.

(18) Beenken, W. J. D.; Pullerits, T., Spectroscopic Units in Conjugated Polymers: A Quantum Chemically Founded Concept?, J. Phys. Chem. B 2004, 108, 6164-6169.

(19) Schwartz, B. J., Conjugated Polymers: What Makes a Chromophore?, Nature Materials 2008, 7, 427-428.

(20) Beenken, W. J. D., Excitons in Conjugated Polymers: Do We Need a Paradigm Change?, Physica Status Solidi a-Applications and Materials Science 2009, 206, 2750-2756.

(21) Lupton, J. M., Chromophores in Conjugated Polymers-All Straight?, Chemphyschem 2012, 13, 901-907.

(22) Healy, A. T.; Boudouris, B. W.; Frisbie, C. D.; Hillmyer, M. A.; Blank, D. A., Intramolecular Exciton Diffusion in Poly(3-Hexylthiophene), J.Phys. Chem. Lett. 2013, 4, 34453449.

(23) Klaerner, G.; Miller, R. D., Polyfluorene Derivatives: Effective Conjugation Lengths from Well-Defined Oligomers, Macromolecules 1998, 31, 2007-2009.

(24) Asaoka, S.; Takeda, N.; Iyoda, T.; Cook, A. R.; Miller, J. R., Electron and Hole Transport to Trap Groups at the Ends of Conjugated Polyfluorenes, J. Am. Chem. Soc. 2008, 130, 1191211920.

(25) Sreearunothai, P.; Asaoka, S.; Cook, A. R.; Miller, J. R., Length and Time Dependent Rates in Diffusion-Controlled Reactions with Conjugated Polymers, J. Phys. Chem. B 2009, 113 27862795.

(26) Wishart, J. F.; Cook, A. R.; Miller, J. R., The Leaf Picosecond Pulse Radiolysis Facility at Brookhaven National Laboratory, Rev. Sci. Instrum. 2004, 75, 4359-4366.

(27) Murov, S. L.; Carmichael, I.; Hug, G. L. Handbook of Photochemistry; 2nd ed.; Marcel Dekker: New York, 1993.

(28) King, S. M.; Cass, M.; Pintani, M.; Coward, C.; Dias, F. B.; Monkman, A. P.; Roberts, M., The Contribution of Triplet-Triplet Annihilation to the Lifetime and Efficiency of Fluorescent Polymer Organic Light Emitting Diodes, J. Appl. Phys. 2011, 109, 074502 1-10. 
(29) Monkman, A. P.; Burrows, H. D.; Hamblett, I.; Navaratnam, S., Intra-Chain Triplet-Triplet Annihilation and Delayed Fluorescence in Soluble Conjugated Polymers, Chem. Phys. Lett. 2001, 340, 467-472.

(30) Burrows, H. D.; de Melo, J. S.; Serpa, C.; Arnaut, L. G.; Miguel, M. D.; Monkman, A. P.; Hamblett, I.; Navaratnam, S., Triplet State Dynamics on Isolated Conjugated Polymer Chains, Chem. Phys. 2002, 285, 3-11.

(31) Frisch, M. J.; Trucks, G. W.; Schlegel, H. B.; Scuseria, G. E.; Robb, M. A.; Cheeseman, J. R.; Jr., J. A. M.; Vreven, T.; Kudin, K. N.; Burant, J. C.et. al.; A.1 ed.; Gaussian Inc.: Pittsburgh, PA, 2003.

(32) Rawashdeh, A. M. M.; Sotiriou-Leventis, C.; Gao, X. R.; Leventis, N., One-Step Synthesis and Redox Properties of Dodecahydro-3a,9a-Diazaperylene - the Most Easily Oxidized PPhenylenediamine, Chem. Commun. 2001, 1742-1743.

(33) Gomes, P. J. S.; Serpa, C.; Arnaut, L. G., (More) About Biphenyl First Excited Triplet State Energy, J. Photochem. Photobiol., A 2006, 184, 228-233.

(34) Streitweiser, A. J.; Schwager, I., J. Phys. Chem. 1962, 66, 2316.

(35) Shalev, H.; Evans, D. H., Solvation of Anion Radicals - Gas-Phase Vs Solution, J. Am. Chem. Soc. 1989, 111, 2667-2674.

(36) Traytak, S. D., The Diffusive Interaction in Diffusion-Limited Reactions - the Steady-State Case, Chem. Phys. Lett. 1992, 197, 247-254.

(37) Tsao, H. K.; Lu, S. Y.; Tseng, C. Y., Rate of Diffusion-Limited Reactions in a Cluster of Spherical Sinks, J. Chem. Phys. 2001, 115, 3827-3833.

(38) Fytas, G.; Nothofer, H. G.; Scherf, U.; Vlassopoulos, D.; Meier, G., Structure and Dynamics of Nondilute Polyfluorene Solutions, Macromolecules 2002, 35, 481-488.

(39) Somma, E.; Loppinet, B.; Chi, C.; Fytas, G.; Wegner, G., Static and Dynamic Solution Properties of Monodisperse Oligofluorenes, Phys. Chem. Chem. Phys. 2006, 8, 2773-2778.

(40) Willard, A. P.; Rossky, P. J.; American Chemical Society: 2013, p PHYS-177.

(41) Mani, T.; Miller, J. R., Role of Bad Dihedral Angles: Methylfluorenes Act as Energy Barriers for Excitons and Polarons of Oligofluorenes, J. Phys. Chem. A 2014, 118, 9451-9459.

(42) Grell, M.; Bradley, D. D. C.; Long, X.; Chamberlain, T.; Inbasekaran, M.; Woo, E. P.; Soliman, M., Chain Geometry, Solution Aggregation and Enhanced Dichroism in the Liquidcrystalline Conjugated Polymer Poly(9,9-Dioctylfluorene), Acta Polymerica 1998, 49, 439444.

(43) Ebihara, Y.; Vacha, M., Relating Conformation and Photophysics in Single Meh-Ppv Chains, J. Phys. Chem. B 2008, 112, 12575-12578.

(44) Sugimoto, T.; Habuchi, S.; Ogino, K.; Vacha, M., Conformation-Related Exciton Localization and Charge-Pair Formation in Polythiophenes: Ensemble and Single-Molecule Study, J. Phys. Chem. B 2009, 113, 12220-12226.

(45) Hu, D. H.; Yu, J.; Wong, K.; Bagchi, B.; Rossky, P. J.; Barbara, P. F., Collapse of Stiff Conjugated Polymers with Chemical Defects into Ordered, Cylindrical Conformations, Nature 2000, 405, 1030-1033. 
TOC Graphic

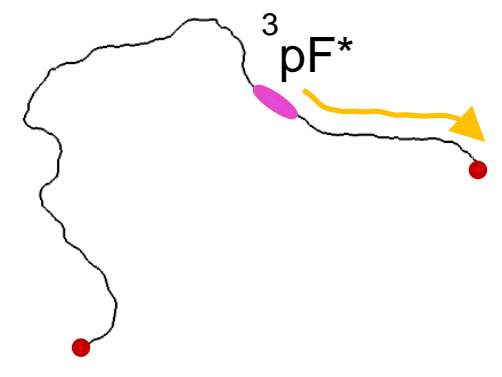




\section{Supporting Information for:}

\section{Continuous Transport of Triplet Excitons Along 100 nm Polyfluorene Chains}

Liang Xi, ${ }^{\dagger}$ Matthew Bird, ${ }^{\dagger}$ Gina Mauro, ${ }^{\dagger}$ Sadayuki Asaoka, ${ }^{\S}$, Andrew R. Cook, ${ }^{\dagger}$ Hung-Cheng Chen, ${ }^{\dagger}$ and John R. Miller, ${ }^{\dagger *}$

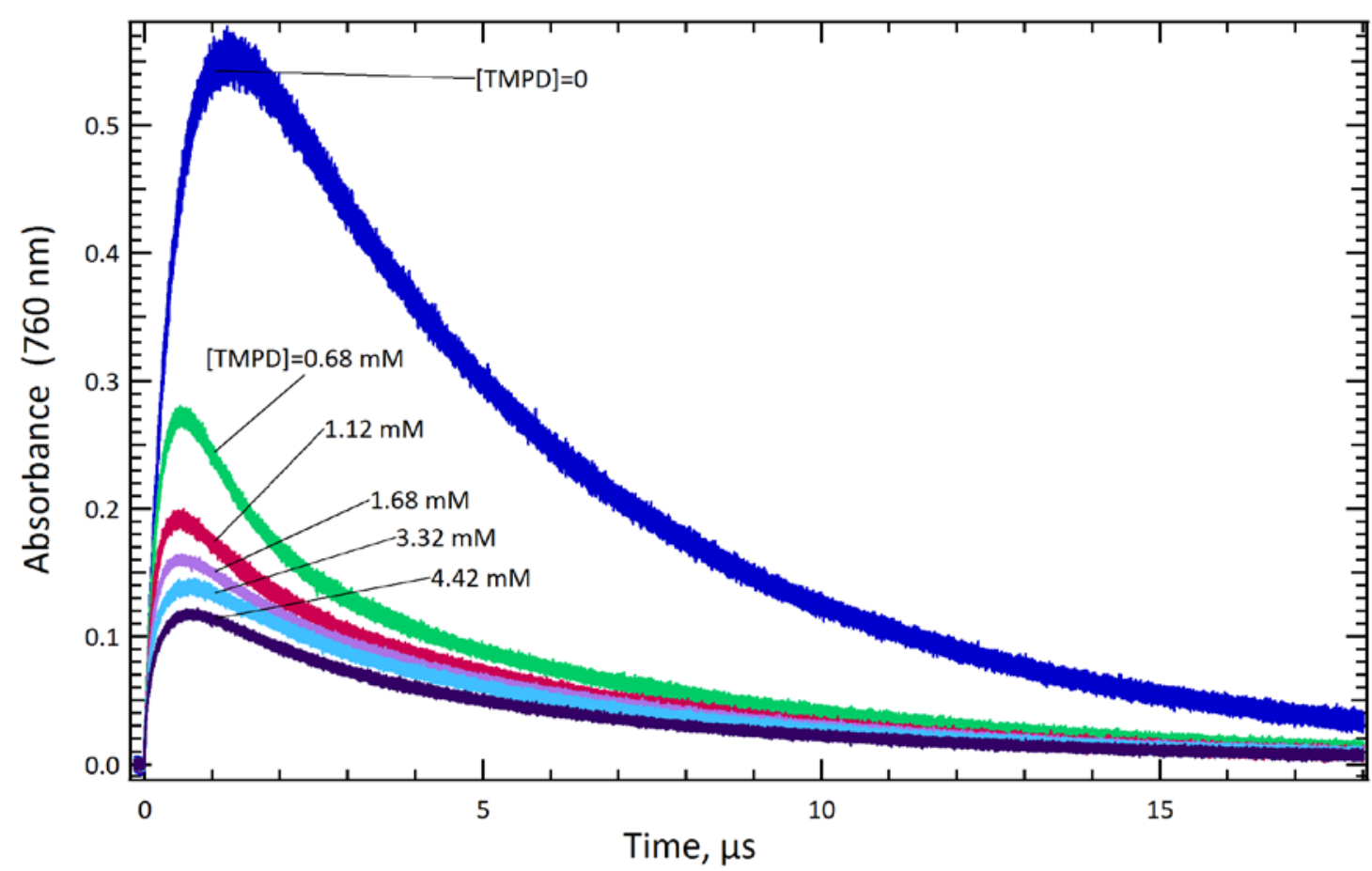

Figure S1 Transient absorbance at the $760 \mathrm{~nm}$ maximum of ${ }^{3} \mathrm{pF}_{18} \mathrm{AQ}^{*}$ created by pulse radiolysis of $100 \mathrm{uM}$ of $\mathrm{pF}_{18} \mathrm{AQ}$ with varied concentrations of TMPD in toluene containing $5 \%$ acetophenone and $50 \mathrm{mM}$ naphthalene. The lowest concentration (0.68 mM TMPD) shows a growth of triplets followed by a $2.4 \mu$ s decay to a long-lived absorption. While not as distinct as in Figure 1 of the main manuscript, the long-lived absorption is decreases with increasing concentration of TMPD, indicating an equilibrium as noted in Figure 1. 


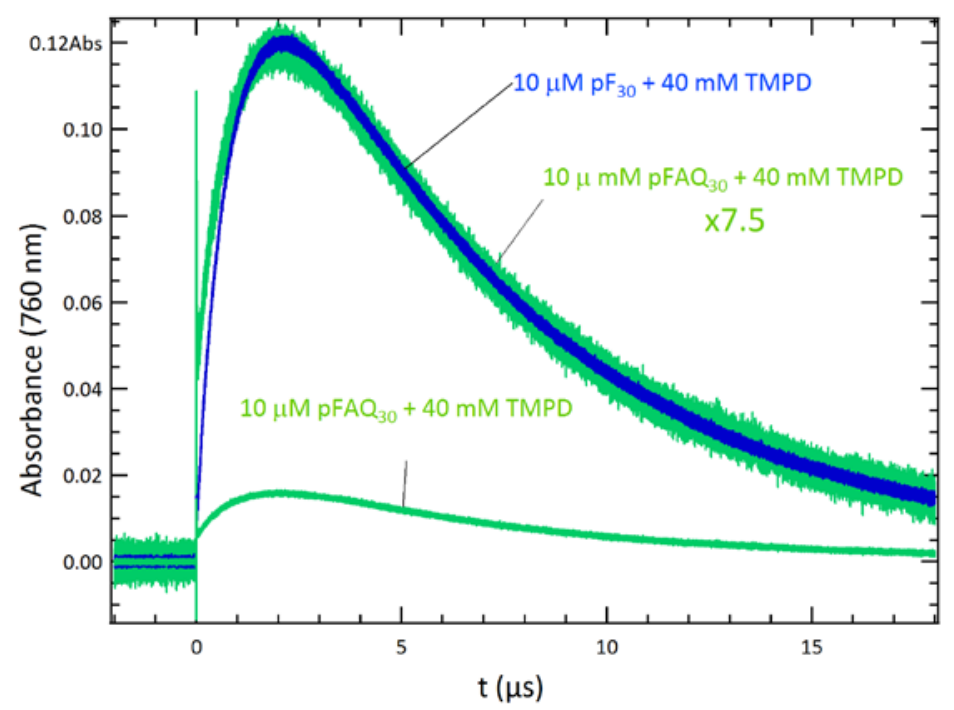

Figure S2 Transient absorbance at the $760 \mathrm{~nm}$ maximum of triplets in $10 \mu \mathrm{M}$ solutions of $\mathrm{pF}_{30}$ or $\mathrm{pF}_{30} \mathrm{AQ}$ in p-xylene containing $40 \mathrm{mM}$ TMPD. The absorbance of the pFAQ trace is smaller by a factor of 7.5 as seen by a scaled version of the same data, indicating that $1 / 7.5=13.3 \%$ of the chains have no AQ end trap groups. At the low, $10 \mu \mathrm{M}$, concentration there is little decay by bimolecular triplet transfer. 
Table S1 Estimates of capping by NMR measurements of end group and side chain resonances, with integrations over length in each fraction. The columns give the fraction label, n(MALS)=the average length in repeat units determined my multi-alngle light scattering, and the probability, $\mathrm{P}_{\text {cap }}$, that a chain end is capped by an NI or AQ trap group, as determined by NMR. From $\mathrm{P}_{\text {cap }}$, the fractions of chains having 0,1 or 2 trap groups were estimated. Average values were calculated from the mean over the shorter fractions , -4 to -9 .

\section{pFNI}

n(MALS) $\quad \mathrm{P}_{\text {cap }} \quad \mathrm{F}_{0} \quad \mathrm{~F}_{1} \quad \mathrm{~F}_{2}$

SAB139-1 117.1

$\begin{array}{llllll}\text { SAB139-2 } & 70.9 & 1.0993 & 0.0099 & -0.2184 & 1.2086 \\ \text { SAB139-3 } & 49.4 & 0.8154 & 0.0341 & 0.3010 & 0.6649 \\ \text { SAB139-4 } & 39.3 & 0.7138 & 0.0819 & 0.4086 & 0.5095 \\ \text { SAB139-5 } & 32.1 & 0.7202 & 0.0783 & 0.4030 & 0.5187 \\ \text { SAB139-6 } & 29.2 & 0.6910 & 0.0955 & 0.4271 & 0.4774 \\ \text { SAB139-7 } & 26.0 & 0.7736 & 0.0513 & 0.3503 & 0.5984 \\ \text { SAB139-8 } & 22.2 & 0.7276 & 0.0742 & 0.3964 & 0.5294 \\ \text { SAB139-9 } & 20.5 & 0.7707 & 0.0526 & 0.3534 & 0.5940 \\ & & 0.7328 & 0.0668 & 0.3771 & 0.5560 \\ \text { AVERAGE } & & & & & \end{array}$

\section{pFAQ}

$\begin{array}{llllll} & \mathbf{n}(\text { MALS }) & \mathrm{P}_{\text {cap }} & \mathrm{F}_{0} & \mathrm{~F}_{1} & \mathrm{~F}_{2} \\ \text { SAB140-1 } & 126.5 & 1.2922 & 0.0854 & -0.7551 & 1.6697 \\ \text { SAB140-2 } & 80.9 & 0.7795 & 0.0486 & 0.3437 & 0.6077 \\ \text { SAB140-3 } & 51.5 & 0.6245 & 0.1410 & 0.4690 & 0.3900 \\ \text { SAB140-4 } & 38.9 & 0.7759 & 0.0502 & 0.3478 & 0.6020 \\ \text { SAB140-5 } & 27.5 & 0.6790 & 0.1030 & 0.4359 & 0.4611 \\ \text { SAB140-6 } & 26.2 & 0.6103 & 0.1519 & 0.4757 & 0.3725 \\ \text { SAB140-7 } & 24.1 & 0.5778 & 0.1782 & 0.4879 & 0.3339 \\ \text { SAB140-8 } & 20.0 & 0.5858 & 0.1716 & 0.4853 & 0.3431 \\ \text { SAB140-9 } & 17.4 & 0.5807 & 0.1758 & 0.4870 & 0.3372\end{array}$

\begin{tabular}{lllll}
\cline { 3 - 4 } AVERAGE & 0.6334 & 0.1275 & 0.4415 & 0.4309
\end{tabular}




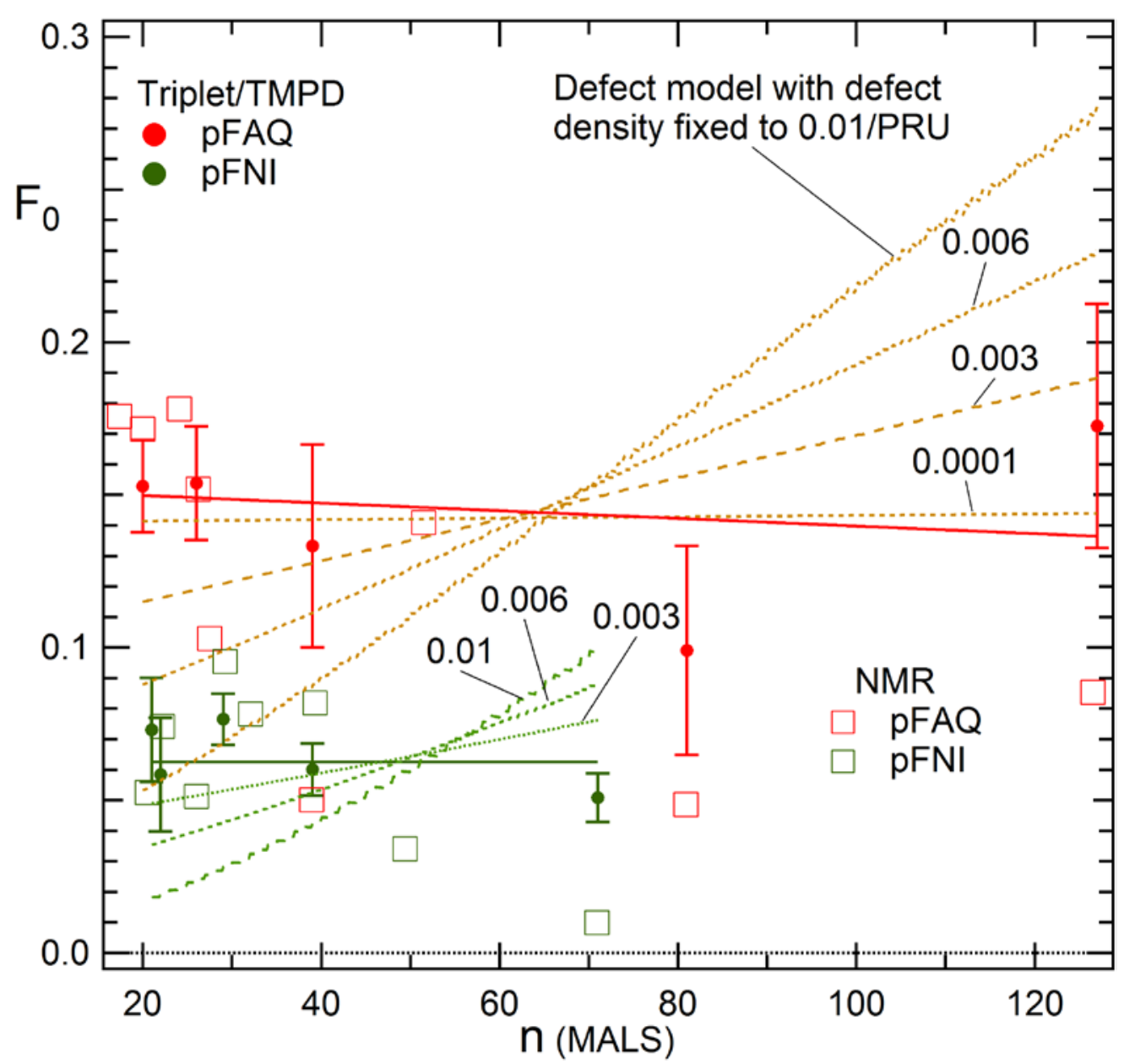

Figure S3 As in Figure 5 fractions of chains having no end trap groups, $\mathrm{F}_{0}$, measured by triplet capture with TMPD (•pFNI, • pFAQ) and by NMR ( $\square$ pFNI, $\square$ pFAQ), as a function of the average lengths of the separated polymer fractions. Also slhown are fits of the triplet/TMPD data to a model that incorporates defects that act as impenetrable barriers to motion of triplet excitons. Those fit lines are for barrier defects present at densities, $\rho$, of 0.001-0.01 per repeat unit. Comparison to this model seems to rule of defect densities $>0.003$ per repeat unit (one defect per 333 repeat units).

The model used in Figure S3 considers a conjugated chain n repeat units long. If each repeat unit is a possible site for a defect and there is a density, $\rho$ per repeat unit, of barrier defects, then the probability of having exactly one defect is given by the probability, $\rho$, of having one defect at a site multiplied by the probability (1-r) ${ }^{(\mathrm{n}-1)}$ that there is no defect on the remaining sites.

$$
\mathrm{P}_{1}=\rho \mathrm{n}(1-\mathrm{r})^{(\mathrm{n}-1)} \quad \mathrm{EqS} 1
$$

Probabilities for having $\mathrm{m}$ defects in the chain are given by similar probabilities and the combinations for arranging the defects.

$$
P_{n}=\left(\rho^{m}\right)(1-\rho)^{(n-m)}\left(n ! /\left(m !^{*}(n-m) !\right) \quad\right. \text { EqS2 }
$$


The model then evaluates the probabilities that the barrier defects prevent excitons from reaching the traps using EqS3 and EqS4, as illustrated in Figure S4 for the case of one barrier defect in chains with two or one end trap group.

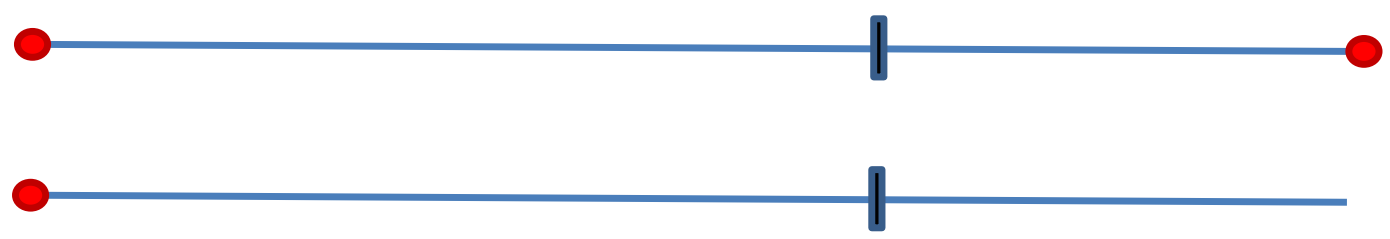

Figure S4 Conjugated chains with a single barrier defect (black) and two (upper) end trap groups. An exciton created anywhere in the chain can reach an end trap. With only one end trap group (lower) a single barrier defect can prevent excitons created in part of the chain from reaching the end trap. Here the barrier leads to survival of about one third of the excitons; on average half would survive. For $m$ randomly placed barrier defects the surviving fractions in chains with one or two end traps would be:

$$
\begin{array}{ll}
F_{\mathrm{s}, 1, \mathrm{~m}}=\mathrm{m} /(\mathrm{m}+1) & \text { EqS3 } \\
\mathrm{F}_{\mathrm{s}, 2, \mathrm{~m}}=\mathrm{m} /(\mathrm{m}+2) & \text { EqS4. }
\end{array}
$$




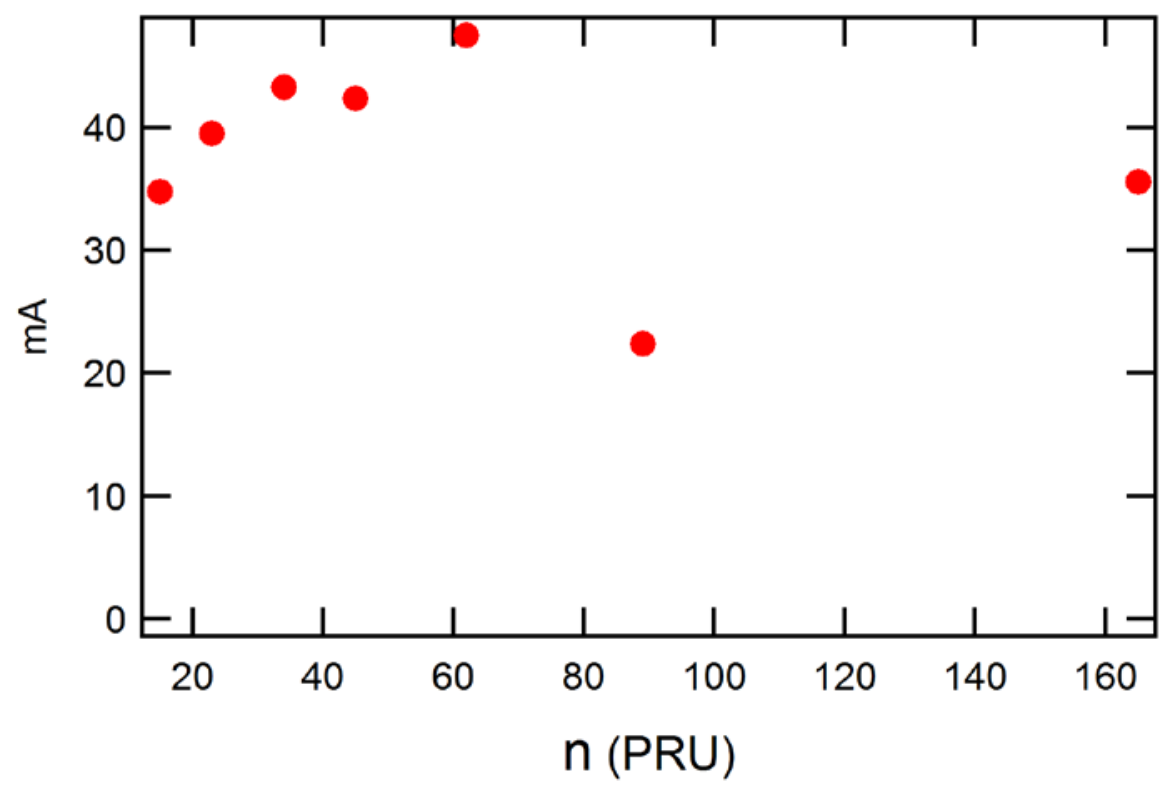

Figure S5 Triplet absorptions of triplets formed in pBOFs having average lengths from 15 to 165 repeat units, each present at $1 \mathrm{mM}$ (PRU) in p-xylene containing $100 \mathrm{mM}$ biphenyl and $10 \mathrm{mM}$ TMPD. 


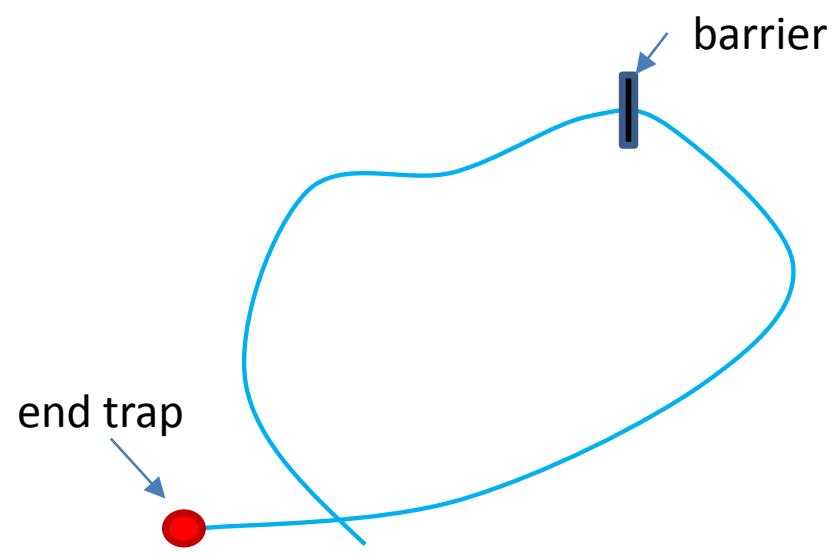

Figure S6 An illustration of a polymer chain with one end trap and a barrier near the middle of the chain. Coiling to make a self-contact, the point where the chains cross, might enable transfer of a triplet from the left side of the chain to the right side so it could then be captured by the end trap.

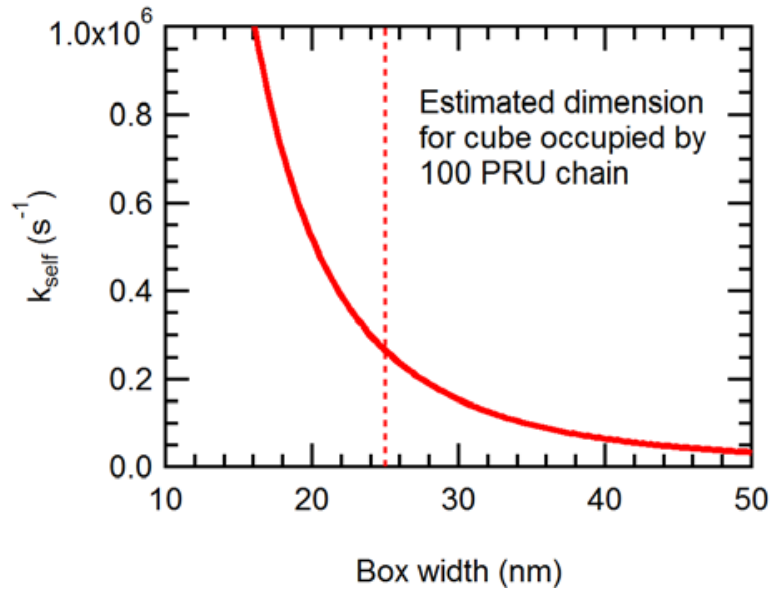

Figure S7 The rate of triplet transfer via self-contacts of 100 PRU long chains from an estimate described below.

Self-contacts estimate: For a chain having e.g. 100 PRU length and one end trap, bending, especially near the middle of the chain, can enable contacts as pictured in Figure S6. Instead, we imagine cutting the chain at the middle to produce two 50 PRU chains that are free to diffuse, but 
required to stay close, by requiring them to remain within a cube. From averaging the volume of a cuboid occupied by simulated ${ }^{1} 100$ PRU chains (1000 averages), we estimate a box of side $25 \mathrm{~nm}$ to be an appropriate size (average volume was found to be $1.6 \times 10^{-17} \mathrm{~cm}^{3}$ ). Dividing by the volume of this cube defines the concentration of the accepting chain. That concentration is multiplied by the rate constant reported in Figure 3 for a 50 PRU chain to give $2.6 \times 10^{5} \mathrm{~s}^{-1}$. This is the point marked with the dashed line in Figure S7. The curve provides estimates of this rate should the box size be changed.

This method is likely to overestimate the rate of self-contacts. Placing chains in small cubes to define a concentration gives contributions from reactions with chains in adjacent cubes that have nothing to do with self-contacts. Here the estimate is used to check the limits on defects. While to most stringent limits on defects come from chains having only one end trap, the effect of defects contained contributions from two or more defects that isolate triplets in segments that are typically near the centers of chains. These triplets would readily react in the cube method of the previous paragraph although they would be less accessible to self-contacts. The estimate above may give an upper limit to the rate of self-contacts.

1. $\quad$ Bird, M. J.; Reid, O. G.; Cook, A. R.; Asaoka, S.; Shibano, Y.; Imahori, H.; Rumbles, G.; Miller, J. R., Mobility of Holes in Oligo- and Polyfluorenes of Defined Lengths. The Journal of Physical Chemistry C 2014, 118, 6100-6109. 